\title{
Microwave Diversity Imaging Using ASAR Approach
}

\author{
Tah-Hsiung Chu* and Chuo-Jan Pan \\ Electrical Engineering Department, \\ National Taiwan University, Taipei, Taiwan, R.O.C. \\ Tel: 886-2-23635251 ext. 541, Fax: 886-2-23638247 \\ E-mail: thc@ew.ee.ntu.edu.tw
}

\begin{abstract}
In this paper the formulation, measurement arrangement and experimental results for microwave diversity imaging of metallic object using ASAR (antenna synthetic aperture radar) approach are presented. In the measurement arrangement, the test object is illuminated by a horn antenna in the near-field range and the object scattered far field is recorded in a multi-frequency and multi-aspect format. The image is reconstructed based on the ASAR approach. Images of three types of metallic objects, including two thin cylinders, a plate and a scaled 1:72 airplane model are presented. Results of simulation and experiment are shown in a good agreement with the scattering object geometry.
\end{abstract}

\section{Introduction}

Microwave diversity imaging of metallic object using multi-frequency and multiaspect technique has been developed for two cases. One is that the scattering object is in the far-field range for the transmitting and receiving antennas [1]. In other words, the scattering object is under a plane wave illumination and the object scattered far field is recorded in a monostatic arrangement. The object image is reconstructed based on the Bojarski's identity. The other case is that the scattering object is in the far-field range of transmitting antenna, while the scattered field is recorded by a linear receiving array in the near-field range [2]. The object image reconstruction is based on the diffraction tomographic approach. In this paper, we present the results of microwave diversity imaging with the scattering object in the near-field of transmitting antenna, and the object scattered far field is recorded from multi-aspect angles. The image reconstruction is based on the ASAR approach [3], which is an imaging approach to identify the locations of secondary scattering off a platform from antenna radiation data.

\section{Formulation}

Figure 1 shows the scattering geometry, in which the scattering object is represented as a set of scattering centers at $P_{,}\left(x_{i}^{\prime}, y_{1}^{\prime}\right)$, and the transmitting antenna is at $\mathrm{A}\left(-r_{d}, 0\right)$. As the transmitting antenna and receiving antenna are both in $z$-polarization, the scatter far field received by an antenna at $B$ is given as

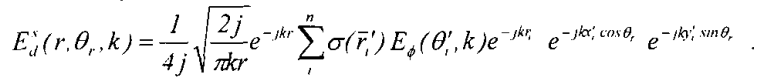

where $E_{\phi}\left(\theta_{r}^{\prime}, k\right)$ is the radiation filed of transmitting horn antenna in the H-plane. $\sigma\left(\bar{r}_{i}\right)$ is the reflectivity of the $i$-th scattering center at $\bar{r}_{1}^{\prime}\left(=\hat{x} x_{t}^{\prime}+\hat{y} y_{t}^{\prime}\right)$. with $r_{i}^{\prime}=\sqrt{\left(x_{i}^{\prime}+r_{d}\right)^{2}+\left(y_{i}^{\prime}\right)^{2}}$ and $\theta_{i}^{\prime}=\tan ^{-1}\left(\frac{y_{i}^{\prime}}{r_{d}+x_{i}^{\prime}}\right) \cdot \hat{r}=-\hat{x} \cos \theta_{r}-\hat{y} \sin \theta_{r}$ is the receiving antenna position. Therefore the range normalized scattered far field becomes 


$$
\begin{aligned}
\Gamma_{d}\left(\theta_{r}, k\right) & =4 j \sqrt{\frac{\pi k r}{2 j}} e^{j k r} E_{d}^{s}\left(r, \theta_{r}, k\right) \\
= & \sum_{i}^{n} \sigma\left(\bar{r}_{i}^{\prime}\right) E_{\phi}\left(\theta_{i}^{\prime}, k\right) e^{-j k r_{r}} e^{-j k x_{i}^{\prime} c u s \theta_{r}} e^{-j k y_{i}^{\prime} s \sin \theta_{r}}
\end{aligned}
$$

As the observation angle is small, and the frequency bandwidth is relatively small to the center frequency, i.e., $k \cos \theta_{r} \cong k, k \sin \theta_{r} \cong k_{0} \theta_{r}$ and $k_{0}$ is wavenumber of center frequency, (2) can be expressed as

$$
\Gamma_{d t}\left(\theta_{r}, k\right) \cong \sum_{i}^{n} \sigma\left(\vec{r}_{i}^{\prime}\right) E_{\phi}\left(\theta_{i}^{\prime}, k\right) e^{-j k\left(r_{i}+x_{i}^{\prime}\right)} e^{-j k_{0} \theta_{r} y_{r}^{\prime}} .
$$

By defining $(u, y)$-space and $\left(p_{u}, p_{y}\right)$-space as $u=r_{i}+x, \quad r_{i}=\sqrt{\left(x+r_{d}\right)^{2}+y^{2}}, p_{u}=k$, $p_{y}=k_{0} \theta_{r}$. (3) then becomes

$$
\Gamma_{d}\left(\theta_{r}, k\right) \cong \sum^{n} \sigma\left(\bar{r}_{r}^{\prime}\right) E_{\phi}\left(\theta_{\prime}^{\prime}, k\right) e^{-t p_{u} u_{r}^{\prime}} e^{-j p_{y} r_{i}^{\prime}} .
$$

Therefore, one can define the scattering object characteristic function as

$$
\gamma_{d}(u, y)=\sum_{i}^{\prime \prime} \sigma\left(\bar{r}_{i}^{\prime}\right) E_{\phi}\left(\theta_{i}^{\prime}, k\right) \delta\left(u-u_{t}^{\prime}\right) \delta\left(y-y_{\prime}^{\prime}\right)
$$

in $(u, y)$-space.

(4) and (5) show that the object scattering function $\gamma_{d}(u, y)$ and range normalized scattered far field $\Gamma_{d f}\left(\theta_{r}, k\right)$ is related by a two-dimensional Fourier transformation as

$$
\operatorname{IFFT}_{2}\left(\Gamma_{d}\left(\theta_{r}, k\right)\right)=\sum_{i}^{n} \sigma\left(\bar{r}_{i}\right) E_{\phi}\left(\theta_{i}^{\prime}, k\right) \delta\left(u-u_{i}^{\prime}\right) \delta\left(y-y_{i}^{\prime}\right)=\gamma_{d}(u, y)
$$

It means that. one can reconstruct the image of scattering object by performing a twodimensional Fourier inversion of $\Gamma_{d}\left(\theta_{r}, k\right)$, then correct the object geometry from (u. y) -space to $(x, y)$-space with $x=\frac{u^{2}-r_{d}{ }^{2}-y_{0}{ }^{2}}{2\left(u+r_{d}\right)}$.

\section{Measurement arrangement and Results}

Figure 2 shows the measurement arrangement developed in our anechoic chamber The test object is illuminated by a Continental Microwave PA42-8 horn antenna at $x=-$ $13 \mathrm{~cm}$. Both the object and transmitting antenna are located on a rotation positioner. The receiving antenna is a Flann Microwave $2024-20$ horn antenna at $x=-150 \mathrm{~cm}$. The object scattered field is measured using a HP8510C network analyzer.

In the measurement, a total of 101 frequency points is swept from $17 \mathrm{GHz}$ to $27 \mathrm{GHz}$, and the aspect angle is from $-14.4^{\circ}$ to $14.4^{\circ}$ with 61 views. A user interactive computer program is developed using HPVEE to control the measurement sequence, calibration and data acquisition.

Three metallic scattering objects are used in the measurement. Figure 3 shows the simulated and measured results of two thin cylinders with $1.75 \mathrm{~cm}$ in radius and $200 \mathrm{~cm}$ in length located at $(6 \mathrm{~cm}, 6 \mathrm{~cm})$ and $(10.5 \mathrm{~cm},-3.5 \mathrm{~cm})$. Figures $3(\mathrm{a})$ and $(\mathrm{b})$ are $\left(p_{i t}, p_{v}\right)$-space data and the reconstructed images are given in Figs. 3 (c) and (d) from simulation and measurement respectively. Both are shown in a good agreement, and the 
reconstructed images gives two bright spots at the correct position of two thin cylinders. Figure 4 are the results of a plate with size $80 \mathrm{~cm}$ by $50 \mathrm{~cm}$ positioned at $19 \mathrm{~cm}$ from the transmitting antenna. Experimental results are shown in good agreement with the simulation results. Figure 5 (a) and (b) are the measured results of a 1:72 scaled airplane model. From the reconstructed image, one can clearly identify the shape and positions of airplane fuselage, engine and oil tanks.

\section{Conclusions}

In this paper, the formulation, measurement arrangement and results of microwave diversity imaging using ASAR approach are presented. It shows that the ASAR approach can not only be used to study the locations of secondary scattering off a platform from the antenna radiation but also to reconstruct the microwave images of a metallic scattering object under a near-field illumination.

References

[1] N. H. Farhat, "Microwave diversity imaging and automated target identification based on models of neural networks," Proc. IEEE, Vol. 77, no.5, pp.670-680, 1989.

[2] D. B. Lin and T. H. Chu, "Bistatic frequency-swept microwave imaging: principle, methodology and experimental results," IEEE Trans. on Antenna and Propaga., Vol. AP-41, pp.25-30, Jan. 1993.

[3] C. Ozdemir, R. Bhalla, L. C. Trintinalia and H. Ling, "ASAR - antenna synthetic aperture radar imaging", IEEE Trans. on Antenna and Propaga., Vol. AP-46, pp.1845-1852, Dec. 1998.
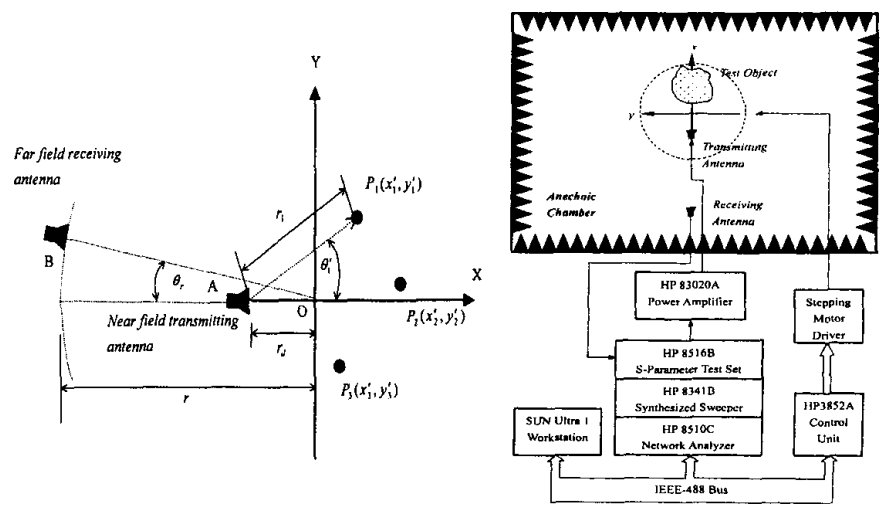

Fig.1 Scattering geometry for microwave diversity imaging with the scattering object in the near-filed range of transmitting horn antenna.

Fig.2 Developed Automated microwave diversity imaging measurement system. 


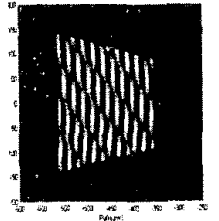

(a)

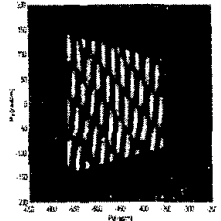

(b)

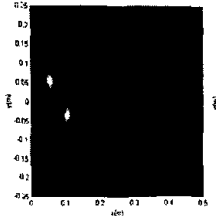

(c)

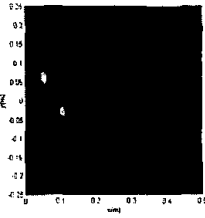

(d)

Fig.3 (a) Simulated and (b) measured results of $\left(p_{u}, p_{y}\right)$-space data and (c) simulated and (d) measured results of reconstructed images for two thin metallic cylinders.

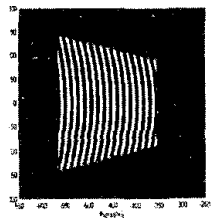

(a)

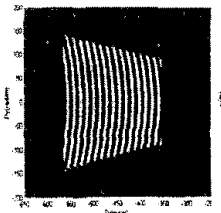

(b)

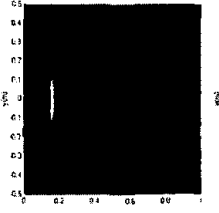

(c)

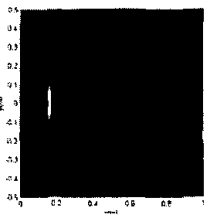

(d)

Fig.4 (a) Simulated and (b) measured results of $\left(p_{u}, p_{y}\right)$-space data and (c) simulated and (d) measured results of reconstructed images for a metallic plate.

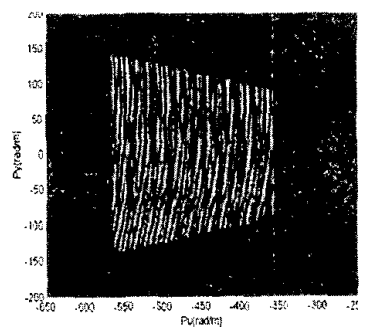

(a)

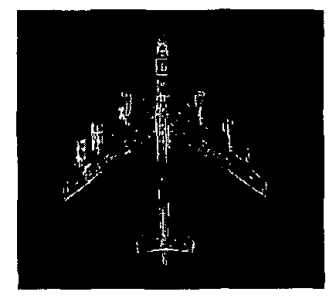

(b)

Fig.5 Measured results of (a) $\left(p_{u}, p_{y}\right)$-space data and (b) reconstructed images for a $1: 72$ scaled airplane model. 